\title{
Offering aggregate results to participants in genomic research: opportunities and challenges
}

\author{
Laura M. Beskow, MPH, PhD1, Wylie Burke, MD, PhD², Stephanie M. Fullerton, D.Phil² and \\ Richard R. Sharp, PhD ${ }^{3,4}$
}

\begin{abstract}
Although issues involved in offering individual results to participants in genomic research have received considerable attention, communication of aggregate results has been the subject of relatively little ethical analysis. Offering participants aggregate results is typically assumed to be a good thing, and studies have found that a significant majority of biobank research participants, when asked about their interest in aggregate results, say that access to such information would be important. Even so, return of aggregate results remains a relatively uncommon practice. In this article, we explore the opportunities involved in communicating aggregate results to participants in genomic research, including affirming the value of research participation, informing participants about research being conducted based on broad
\end{abstract}

\section{INTRODUCTION}

Although issues involved in offering individual results to participants in genomic research have received considerable attention, ${ }^{1-3}$ communication of aggregate results has been the subject of relatively little ethical analysis. Offering participants aggregate results-the overall study findings or conclusions drawn from the population of research participants-is typically assumed to be a good thing. For instance, guidelines on reporting genetic research results to study participants issued by a National Heart, Lung, and Blood Institute working group ${ }^{4}$ suggest that aggregate results should always be returned. The European Union's Organisation for Economic Co-operation and Development ${ }^{5}$ recommends that "summary results arising from research conducted using [human biobank and genetic research database] resources should be made available in easily accessible forms, such as through a newsletter or website" (p. 203). An influential report from the Rand Corporation ${ }^{6}$ simi- $^{-}$ larly concluded that "a best practice for repositories is to provide feedback to tissue sources and physicians about generalized findings from research with repository resources, through the Internet, sessions at scientific meetings, newsletters, or other outreach venues." Certainly, studies have shown that when prospective biobank participants are asked about their interest in aggregate results, a significant majority say that access to such information would be important. ${ }^{7,8}$

Even so, return of aggregate results remains a relatively uncommon practice. ${ }^{2,6}$ Studies of biobank participants' reactions to actual communication of aggregate results are lack- consent for future unspecified research, educating participants and the public about the research process, and building trust in the research enterprise. We also explore some of the challenges, including the complex intersection between individual and aggregate results, as well as practical hurdles. We conclude by offering our preliminary recommendations concerning the provision of aggregate results and an agenda for much-needed future research.

Genet Med 2012:14(4):490-496

Key Words: aggregate results; education; informed consent; research participants; research results; trust

ing, ${ }^{4}$ and there is little evidence or consensus regarding the best approaches to offering such results. ${ }^{9}$

In this article, we explore the opportunities for strengthening the research process that are made possible by communication of aggregate results to participants in genomic research. We also explore some of the practical challenges involved, as well as the complex intersection between returning aggregate findings, participants' perceptions of the implications of the findings for themselves, and researchers' continuing obligations with regard to individual results. We conclude by offering our recommendations concerning the provision of aggregate results and an agenda for much-needed future research.

\section{OPPORTUNITIES IN OFFERING AGGREGATE RESULTS}

Discussions about returning genomic research results are often framed in terms of personal benefits or risks to participants. Debates tend to focus on individual results and competing claims about the obligations researchers may have to provide results-e.g., because of associated health-care benefits that receipt of results may impart to participants ${ }^{4,10-12}$ - and the potential for harm or misunderstanding associated with providing provisional research data. ${ }^{13,14}$ Framing return of results only around individual interests, however, misses the opportunities afforded by offering participants information about the overall study findings. Providing aggregate results, although not a substitute for meeting obligations concerning individual results, can be a manifest recognition of participants' altruism

${ }^{1}$ Center for Genome Ethics, Law, and Policy, Duke Institute for Genome Sciences and Policy, Durham, North Carolina, USA; ${ }^{2}$ Department of Bioethics and Humanities, University of Washington, Seattle, Washington, USA; ${ }^{3}$ Department of Bioethics and Genomic Medicine Institute, Cleveland Clinic, Cleveland, Ohio, USA; ${ }^{4} \mathrm{Center}$ for Genetic Research Ethics and Law, Case Western Reserve University, Cleveland, Ohio, USA. Correspondence: Laura M. Beskow (laura.beskow@duke.edu) 
and perhaps satisfy the curiosity of participants who are eager to know whether their contribution helped to address important scientific questions. Providing aggregate results also serves to convey information about the research actually conducted when this was not specified during the initial informed consent process and may help to demystify an otherwise largely inscrutable research process for participants and the public alike. Together, these effects have the potential to increase trust in the research enterprise, with implications for recruitment, retention, and public support for genomic research more broadly.

\section{Affirming the value of research participation}

From the perspective of relational ethics, ${ }^{15,16}$ offering information about the outcomes of genomic research to participants is a way of demonstrating respect and reciprocity-an expression of gratitude for their charity in providing their biological materials and personal information that make research possible.

The rationale for offering aggregate results as a matter of "recognition" is an easy case to make for research studies or repositories that involve ongoing contact between researchers and participants. For example, the National Center for Healthcare Ethics argued that it is especially appropriate to share aggregate results of projects that make onerous or intimate demands on participants, or where participants are involved through multiple contacts or for an extended time. ${ }^{9}$ Not coincidentally, ongoing communication of aggregate findings is a prominent feature of community-based participatory research, where research volunteers are engaged as partners in the advancement of science. ${ }^{17,18}$

Even in research contexts involving less intensive engagement, however, offering aggregate results could be a powerful and effective means of letting participants know that their contribution is valued. In one study of a proposed biobank, for example, over two-thirds of interviewees said it would be important or very important to receive general news about studies being done using stored specimens and data. ${ }^{7}$ The most frequently mentioned motivations were a desire to stay updated about studies being conducted and to know the outcome or contribution of their participation (e.g., whether their samples were put to "good use"); other common reasons included themes of reciprocity, mutual benefit, and the perception of aggregate results as a kind of "compensation." ${ }^{19}$ Communities participating in the International Haplotype Map project also expressed a strong interest in knowing how samples would be used; this feedback led to procedures to ensure that investigators reported on study goals, as well as quarterly publication of sample use and resulting publications. ${ }^{20}$ The Marshfield Clinic, responding to similar requests from the community advisory group for its population-based biobank, has utilized a twice-yearly newsletter, community talks, and media coverage to provide information about biobank-related research. ${ }^{21}$

Some researchers may view participant interest in knowing about sample use and research outcomes as a burden because of the time and other resources needed to prepare materials that are easily accessible to audiences with varying levels of scientific knowledge. However, participant interest could also be viewed as an indicator that participants perceive a stake in the research process; ${ }^{22}$ thus, efforts to provide information have the potential to kindle increased willingness to support and participate in research.

In addition, it is possible that offering aggregate results could sometimes fulfill participants' stated interest in receiving individual results. We are not suggesting that return of aggregate results obviates any obligation researchers may have to offer individual results. However, when participants say they want results because they would like to know what researchers learned, providing aggregate results may be sufficient to satisfy this commonly stated interest. ${ }^{19,22}$ Information about how their participation contributed to scientific progress may provide participants with the feedback they are seeking; it also represents a perceptible giving back, particularly when individual results are too provisional to provide meaningful information. This is, however, an empirical question; research is needed to determine whether and in what situations general information about study outcomes might adequately address participants' interest in receiving results.

\section{Providing information beyond broad initial consent}

For many biobanks, the storage and widespread sharing of biospecimens and data make it impossible to describe in detail or even to foresee all of the future research for which these materials might be used at the time they are collected. ${ }^{23}$ The participant experience often involves an initial research interaction where biological specimens and various forms of physiological, clinical, and/or self-reported data are collected. Although participants may have high hopes for the kinds of research advances that will be enabled by their contribution, they typically sign a broadly worded consent form and thus may have very little practical idea of the types of questions that will be asked, and answered, using stored specimens and data. Instead, they trust that researchers will use the materials wisely and hope that someone may eventually benefit.

Although some question the sufficiency of this kind of broad consent, ${ }^{24-26}$ others suggest that such consent is acceptable if two conditions are fulfilled: the approval of future projects by a research ethics committee and participants' continuing right to discontinue participation at any time..$^{27,28}$ Offering aggregate research results may help address some of these concerns and conditions by letting participants know whether and how their contributions are serving social goals they support and by making participants' opportunity to withdraw "real."23 In other words, access to aggregate results would provide participants a mechanism by which they can learn about the research actually being conducted-without which the option of withdrawing if they object to the research is relatively meaningless.

Studies have documented some participants' keen interest in knowing how specimens or data stored in a repository might be used and by whom ${ }^{29-31}$ and suggested that certain uses may generate mixed feelings. For instance, in focus groups on data sharing, some participants expressed concern about sharing data with pharmaceutical companies or other entities that 
would result in participants' altruistic contributions being used to generate private profits. However, participants in the same discussions also acknowledged that new drug development reflected a successful outcome for health research. ${ }^{29,31}$ Thus, providing descriptions of the kinds of studies being conducted and the kinds of researchers who are accessing stored materials may fulfill an important informational need, in addition to the return of aggregate study findings.

Finally, offering aggregate results may serve as a useful first step in the longer term process of communicating individual research findings. Return of aggregate results can alert participants to the possibility of new findings and encourage those who may be interested in learning more to reexamine their personal preferences about receiving individual results. Where appropriate, this approach could remind participants of a prospect that may have originally appeared unlikely and reconnect them with investigators. This may be especially important in circumstances where preferences about the receipt of individual results-which participants may have been asked to express at the time of initial consent-may have changed over time.

\section{Education about the research process}

Disseminating aggregate results could serve as an important educational tool for research participants and for the general public. Although many people have high expectations for biomedical research, they often have a limited understanding of the incremental steps involved in generating new knowledge. One widely underestimated aspect of the research process is the amount of time required to translate a specific research observation into generalizable knowledge of sufficient validity and reliability to inform a medical test or other health intervention. ${ }^{32,33}$ Routine dissemination of aggregate results from studies of stored biospecimens and data could help to demystify the process, particularly if the information is provided in a format and language accessible to audiences with a range of scientific literacy and placed within an overall context of research progress. Repositories might even want to borrow from marketing strategies that promote customer loyalty or "stickiness," ${ }^{34}$ including features such as general information about current research, opportunities to ask questions and give feedback, and/or hosting of online interactive discussions.

\section{Building trust in the research enterprise}

Ultimately, scientists who create and maintain genomic biobanks are stewards of those materials and the trust that is essential to their continued existence. Like all researchers, genomic scientists should consider how their interactions with participantseven those research volunteers that they may never meet in person-can affect trust in biomedical research as a whole and the institutions engaged in this work. That trust will become even more critical to the long-term sustainability of biobanks that aim to support a variety of research through the ongoing collection of detailed medical and health information as well as those that plan to share stored materials with a diverse array of distant secondary investigators who are unknown to participants.
Regular communication of aggregate results could foster participant trust in genomic research by reporting outcomes, explaining how a particular study is positioned in the translational pathway, and potentially soliciting participant feedback on questions of importance to effective research (e.g., procedures intended to foster recruitment and retention). Regular updates about research progress-including the inevitable setbacks and challenges-can help to forge the types of relationships that are critical to informed trust in the research enterprise.

Using dissemination strategies that are accessible to the general public, as well as participants, can provide the added value of enhancing public interest in research and demonstrating trustworthy practices. For example, biobanks could create a website and possibly even make use of new forms of social networking to communicate about research accomplished using stored materials. Although public trust is affected by many considerations that fall outside the scope of an individual investigator's control, long-term confidence in the integrity of research requires that members of the public appreciate both the aspirations and limitations of biomedical research. ${ }^{35}$

\section{CHALLENGES IN OFFERING AGGREGATE RESULTS}

The above-mentioned considerations highlight the need for ongoing, bidirectional communication with participants about the aggregate results of genomic research. Through such communication, investigators demonstrate respect for the contributions of research volunteers, respond to participants' interests in receiving information about research outcomes and the research enterprise in general, and create an opportunity for both participant and public feedback. These measures promote the type of informed public trust that is essential to the longterm success of genomic research. Achieving these aims can be more difficult than it might appear, however, and there are a number of challenges that must be overcome for investigators to make aggregate results available to interested participants.

One significant challenge is that aggregate results may have individual implications and thus provoke a wide range of reactions in individuals-from intellectual interest without a particular assignment of personal meaning to surprise, anger, fear, or anxiety with regard to his or her individual status relative to the overall findings. In some cases, sharing aggregate results could lead to negative psychosocial repercussions and possibly create unintended demand for individual results that otherwise do not meet agreed-upon thresholds for disclosure.

As noted, there is little empirical evidence regarding biobank participants' reactions to actual communication of aggregate results. However, studies in the context of epidemiologic research indicate that some of these concerns may be warranted. For example, Bunin and colleagues ${ }^{36}$ sent a letter to parents who participated in a case-control study of pediatric brain tumors summarizing the results of the study, along with a questionnaire. Respondents rated the information as both important and clear, although nearly half commented on the inconclusiveness of the findings. The authors characterized respondents' comments about this as including resignation, 
sadness, surprise, frustration, or anger. More patient mothers than control mothers felt that the results applied to their child, and patient mothers were more likely to want additional information and a phone number to call with questions.

In a clinical trial setting, Dixon-Woods and colleagues interviewed participants about their responses to receiving a summary of the results of a study of antibiotics for preterm labor. ${ }^{37}$ Reactions to the summary were generally positive or neutral; half of the women found the information clear, and half expressed feelings of pleasure on receiving the information, including feeling that taking part had been worthwhile. However, several pointed to particular parts of the summary that were difficult to understand, and several found it difficult to understand in general. Many attempted to interpret the results in terms of their own pregnancy, and others were disappointed that the trial had not revealed the causes of preterm birth and treatments to prevent it. These observations led the authors to conclude, "Providing results to participants in research studies is not straightforward; it constitutes an intervention in its own right and requires more rigorous evaluation than it has previously received." ${ }^{37}$

These kinds of outcomes suggest that when aggregate results are returned, attention should be paid both to the nature of the results and to the way they are communicated. Researchers will need to consider the implications of the findings for individuals as well as the overall contribution to scientific knowledge in crafting the message describing the aggregate results (Table 1 ). When results have little significance for individuals, that message may be fairly straightforward. When results have potential implications for individual participants, however, researchers will need to consider whether they have an ethical obligation to return individual as well as aggregate results. Such an obligation could depend on a variety of contextual factors, including intensity and duration of researcher-participant relationships and the degree of participant vulnerability to and dependence on researchers. ${ }^{38}$ In general, available guidelines for the return of individual research results or incidental findings suggest that results that are provisional or inconclusive should not be returned. ${ }^{4,39,40}$ In contrast, summaries of aggregate results might be accompanied by an explanation of the additional research needed to validate the results and to understand their implications for disease risk, inheritance, diagnosis, prognosis, and treatment. ${ }^{41}$ Again, this type of explanation provides an opportunity to increase understanding of the incremental nature of research, potentially shedding light on what is for many a foreign and unfamiliar activity.

Although there is potential for unanticipated harm, these possibilities do not justify withholding aggregate results. "Protecting" people from information that may be of personal interest or that may be seen as relevant to their health is neither feasible nor defensible, given that such information-including reports about research discoveries-is broadly available from many sources to participants and nonparticipants alike. Instead, the challenges posed by return of aggregate results point to important ethical considerations about how they are communicated.
To share aggregate results responsibly, a number of practical impediments must be addressed. First, time and resources must be dedicated to preparing and disseminating a lay summary of aggregate results.,92 This may include obtaining institutional review board input on the plans and materials. Second, the potential impact on other stakeholders must be considered, including the possible burden on treating physicians if the aggregate results generate significant questions or confusion. Investigators must be sensitive to this possible blurring between research and medical care, and emphasize the research context in their communication of aggregate results. Achieving this goal may be easier if aggregate results are combined with general information about research, including explanations of what is known now, both from the study and other research, and what remains to be determined. Finally, many empirical questions remain about the communication process itself, such as which dissemination strategies are most effective; defining optimal approaches is a critical topic for further study in the research context, as it is more generally in public health. ${ }^{43}$

\section{CONCLUSIONS AND FUTURE DIRECTIONS}

Offering aggregate results of genomic research is an important way to demonstrate respect for persons and gratitude for their help in generating the data needed to address a study's research questions. ${ }^{44}$ Wide dissemination of aggregate research results in lay language brings a number of crucial benefits to researchers, participants, and the public. We argue that these benefits outweigh any concerns about such dissemination. Communicating aggregate results to biobank participants may not rise to the level of an ethical obligation, but it should be considered good research practice and the default expectation. A researcher who believes dissemination of aggregate results is not possible should be prepared to justify this position to institutional review boards, funding agencies, and the general public.

The primary justifications for not offering aggregate results are cost and feasibility. Currently, there are a number of issues related to the availability of resources to communicate results. Aggregate results may not be fully known until years after the close of a research study, for example. The financial resources for the study may have ended by that time, with limited options available to investigators wishing to return key findings to research volunteers. In addition, it may be unclear how best to communicate results. In the context of research linked to biorepository collections, some of these concerns may be addressed by biobanks' adoption of explicit communication strategies to inform participants and the public about biobank-enabled discoveries. In other cases, concerns must be addressed at the research system level (either by the institution with which the biobank is affiliated or perhaps by national funding agencies or similar), not seen as a responsibility solely of investigators. It is against this background that we offer the following recommendations as well as an agenda for future research. 
Table 1 Implications of research topic and message to participants: hypothetical examples

\begin{tabular}{lll} 
Study & Main findings & Implications for participants \\
\hline $\begin{array}{l}\text { GWAS to identify gene variants } \\
\text { associated with height }\end{array}$ & $\begin{array}{l}\text { Multiple common gene } \\
\text { variants identified, each with } \\
\text { small effect; findings together } \\
\text { explain only a small portion of } \\
\text { heritability of height }\end{array}$ & $\begin{array}{l}\text { No medical significance; results } \\
\text { unlikely to generate anxiety }\end{array}$ \\
\hline $\begin{array}{lll}\text { GWAS to identify gene variants } \\
\text { associated with T2DM }\end{array}$ & $\begin{array}{l}\text { Multiple common gene } \\
\text { variants identified, each with } \\
\text { small effect; findings together } \\
\text { explain 20\% of heritability of } \\
\text { T2DM }\end{array}$ & $\begin{array}{l}\text { Participants (particularly those not } \\
\text { currently affected by T2DM but } \\
\text { who may be at increased risk of } \\
\text { future disease) may be interested in } \\
\text { personal results }\end{array}$ \\
\hline
\end{tabular}

\begin{abstract}
Main message in return of aggregate results

Provide clear explanation of small effect of identified variants, and likelihood that other genetic factors will be found. Explain how this knowledge contributes to better scientific understanding of genetic contribution to physical traits.
\end{abstract}

Provide clear explanation of small effect of variants; in addition, explain preventive measures for T2DM and that current medical recommendations would not change based on results. Explain next steps, e.g., to determine whether genetic information would have clinical utility if added to established risk assessment techniques such as family history.

\section{Study suggests moderate positive predictive value and high negative predictive value of genotype for adverse drug reaction \\ Participants may wish to know their genetic status for use in clinical care; however, clinical utility of the information for study participants is low because they have already been treated and their reaction status is known}

Gene and causative mutations identified
Some family members, in particular, sisters of affected, may benefit from testing based on study results, to determine whether they are carriers for the disorder
As relevant, explain need to replicate and need to develop test protocol for clinical lab, prior to clinical use. Also, explain limited relevance to study participants. This study represents an example where researcher may consider returning individual results, depending on status of test validation and potential to inform future drug prescribing.

Explain results, emphasizing that they do not have any immediate treatment implications but will assist researchers to understand the disease better, with potential clinical benefits in the future (likely a long time lag). This is an example where researchers are likely also to feel an obligation to return individual results.

GWAS, genome-wide association study; T2DM, type 2 diabetes mellitus.

\section{Recommendations}

1. Researchers should communicate clearly with participants and the public about research conducted and its outcomes. As a matter of transparency and accountability, biobanks and genomic researchers should devise ways to communicate clearly with participants and the general public about the research being conducted and what is being learned. ${ }^{7}$ This communication is important even when the results are inconclusive or do not have potential health implications for research participants.

2. Results should be made as accessible as possible and communicated in lay language that identifies both major findings and study limitations. Summaries of research results should be available in lay language and emphasize the "research" (provisional) nature of the results in order to minimize confusion and therapeutic misconception. Secondary researchers' responsibilities to provide the biobank with such a summary should be made clear (e.g., in material transfer agreements). Biobanks and genomic researchers should work with health communications experts and representatives of the biobank population whenever possible to develop such summaries. Links could also be provided to scientific literature for those who are interested.
3. Funding agencies should support the return of aggregate results as matter of good research practice. Research funders should support the communication of aggregate results to participants and the general public as a matter of good research practice. Investigators should also strive to include personnel and financial resources to facilitate these activities in their requests for research support. To the extent that such support may be difficult to arrange using traditional funding mechanisms, funders should develop supplemental support mechanisms that can accommodate the more extended timelines associated with return of aggregate results.

4. Moving forward, plans for return of aggregate results should be described in grant applications and institutional review board protocols and should be disclosed at the time consent is sought from research participants. Plans for the communication of aggregate results should be described in institutional review board protocols and disclosed during the informed consent process. In circumstances where the optimal communication mechanism is not known at the outset of the study, researchers should still allow for this possibility in study protocols, potentially including measures and resources to seek input from participants regarding their preferences. 
Table 2 Key research questions for communication of aggregate results

- How: Effectiveness, feasibility, financial costs, and participant satisfaction associated with various communication methods (e.g., newsletters, websites, community presentations, media coverage, social networking tools) for different study populations and study designs

- What: Which aspects of aggregate results to focus on (e.g., in the context of biobanks, should communication include general descriptions of how stored materials were used, by whom, and for what kinds of studies; communication of results from individual studies; or both? Should participants be able to access information about the specific studies in which their samples and data were actually used? What additional information would participants or the general public like to have?)

- When: When to report findings relative to scientific publication/dissemination; need for updates as more knowledge accumulates

- Who: Responsibilities for preparing and communicating aggregate result summaries when researcher has no direct relationship with participants

5. Return of aggregate results should not be used as a way to avoid an obligation to offer individual findings. If the information generated from research meets the accepted threshold for return of individual results, ${ }^{4,12,39}$ researchers should not return aggregate results alone with the expectation that participants will recognize the potential personal importance and follow up on their own.

6. National policies should be developed to promote the routine and widespread communication of aggregate results, particularly in the context of large, highly visible, publicly funded studies. National policy should be developed to address the roles and responsibilities of biobanks-particularly large population-based efforts that produce findings of potential importance to public health and larger amalgamated collections (e.g., the database of Genotypes and Phenotypes) that are many steps removed from the point of initial recruitment and consent-with regard to the return of aggregate results and especially the communication of findings to the public at large.

We also suggest the following points to consider that may apply in particular circumstances and that would benefit from further discussion and dialog:

- If aggregate results are conveyed actively (e.g., via direct mailing), participants should be given a choice about receiving such results at the time that they consent to participate.

- Making aggregate results of limited significance easily yet passively available (e.g., via a website) may be a way to avoid the appearance of assigning unwarranted importance or creating confusion among research participants regarding the salience of the results.

\section{Future research agenda}

Implementing these recommendations will not be straightforward. ${ }^{42}$ Research is needed (Table 2) to better understand participant and researcher needs and preferences, systematically assess the positive and negative outcomes of sharing such results, develop strategies for increasing participant understanding of aggregate results from genomic research, and develop best practices by which results can be shared responsibly.

\section{ACKNOWLEDGMENTS}

This work was supported by grants R01-HG003178 to the University of Minnesota, UL1-RR024128 to Duke University, P50-
HG3374 and U01-HG004610 to the University of Washington, and P50-HG003390 to Case Western Reserve University. The views expressed are those of the authors and do not necessarily reflect the views of the funding agencies that provided support or the institutions with which the authors are affiliated.

\section{DISCLOSURE}

The authors declare no conflict of interest.

\section{REFERENCES}

1. Haga SB, Beskow LM. Ethical, legal, and social implications of biobanks for genetics research. Adv Genet 2008;60:505-544.

2. Dressler LG. Disclosure of research results from cancer genomic studies: state of the science. Clin Cancer Res 2009;15:4270-4276.

3. Bredenoord AL, Kroes HY, Cuppen E, Parker M, van Delden JJ. Disclosure of individual genetic data to research participants: the debate reconsidered. Trends Genet 2011;27:41-47

4. Fabsitz RR, McGuire A, Sharp RR, et al.; National Heart, Lung, and Blood Institute working group. Ethical and practical guidelines for reporting genetic research results to study participants: updated guidelines from a National Heart, Lung, and Blood Institute working group. Circ Cardiovasc Genet 2010;3:574-580.

5. Organisation for Economic Cooperation and Development. OECD guidelines on human biobanks and genetic research databases. Eur J Health Law 2010;17:191-204.

6. Eiseman E, Bloom G, Brower J, Clancy N, Olmsted SS. Case Studies of Existing Human Tissue Repositories. "Best Practices" for a Biospecimen Resource for the Genomic and Proteomic Era. RAND Corporation: Santa Monica, CA, 2003.

7. Beskow LM, Dean E. Informed consent for biorepositories: assessing prospective participants' understanding and opinions. Cancer Epidemiol Biomarkers Prev 2008;17:1440-1451.

8. Meulenkamp TM, Gevers SK, Bovenberg JA, Koppelman GH, van Hylckama Vlieg A, Smets EM. Communication of biobanks' research results: what do (potential) participants want? Am J Med Genet A 2010;152A:2482-2492.

9. US Department of Veterans Affairs. Sharing Aggregate Research Results With Participants. http://www.ethics.va.gov/docs/infocus/InFocus_20090330_ Sharing_aggregate_research_results.pdf (2009).

10. Fernandez CV, Kodish E, Weijer C. Informing study participants of research results: an ethical imperative. IRB 2003;25:12-19.

11. Knoppers BM, Joly Y, Simard J, Durocher F. The emergence of an ethical duty to disclose genetic research results: international perspectives. Eur J Hum Genet 2006;14:1170-1178.

12. Wolf SM, Lawrenz FP, Nelson CA, et al. Managing incidental findings in human subjects research: analysis and recommendations. J Law Med Ethics 2008;36:219-248, 211.

13. Parker LS. Best laid plans for offering results go awry. Am J Bioeth 2006;6: 22-23; author reply W10.

14. Miller FA, Christensen R, Giacomini M, Robert JS. Duty to disclose what? Querying the putative obligation to return research results to participants. J Med Ethics 2008;34:210-213.

15. Goering S, Holland S, Fryer-Edwards K. Transforming genetic research practices with marginalized communities: a case for responsive justice. Hastings Cent Rep 2008;38:43-53.

16. King NMP, Henderson GE, Stein J. Beyond Regulations: Ethics in Human Subjects Research, 1st edn. University of North Carolina Press: Chapel Hill, NC, 1999. 
17. Israel BA, Schulz AJ, Parker EA, Becker AB. Review of community-based research: assessing partnership approaches to improve public health. Annu Rev Public Health 1998;19:173-202.

18. Chen PG, Diaz N, Lucas G, Rosenthal MS. Dissemination of results in communitybased participatory research. Am J Prev Med 2010;39:372-378.

19. Beskow LM, Smolek SJ. Prospective biorepository participants' perspectives on access to research results. J Empir Res Hum Res Ethics 2009;4:99-111.

20. Rotimi C, Leppert M, Matsuda I, et al.; International HapMap Consortium. Community engagement and informed consent in the International HapMap project. Community Genet 2007;10:186-198.

21. McCarty CA, Chapman-Stone D, Derfus T, Giampietro PF, Fost N; Marshfield Clinic PMRP Community Advisory Group. Community consultation and communication for a population-based DNA biobank: the Marshfield clinic personalized medicine research project. Am J Med Genet A 2008;146A:3026-3033.

22. Trinidad SB, Fullerton SM, Ludman EJ, Jarvik GP, Larson EB, Burke W. Research ethics. Research practice and participant preferences: the growing gulf. Science 2011;331:287-288.

23. McGuire AL, Beskow LM. Informed consent in genomics and genetic research. Annu Rev Genomics Hum Genet 2010;11:361-381.

24. Caulfield T. Gene banks and blanket consent. Nat Rev Genet 2002;3:577.

25. Greely HT. The uneasy ethical and legal underpinnings of large-scale genomic biobanks. Annu Rev Genomics Hum Genet 2007;8:343-364.

26. Mascalzoni D, Hicks A, Pramstaller P, Wjst M. Informed consent in the genomics era. PLoS Med 2008;5:e192.

27. Elger BS, Caplan AL. Consent and anonymization in research involving biobanks: differing terms and norms present serious barriers to an international framework. EMBO Rep 2006; 7:661-666.

28. O'Doherty KC, Burgess MM, Edwards K, et al. From consent to institutions: designing adaptive governance for genomic biobanks. Soc Sci Med 2011;73:367-374.

29. Trinidad SB, Fullerton SM, Bares JM, Jarvik GP, Larson EB, Burke W. Genomic research and wide data sharing: views of prospective participants. Genet Med 2010;12:486-495.
30. Ludman EJ, Fullerton SM, Spangler L, et al. Glad you asked: participants' opinions of re-consent for dbGap data submission. J Empir Res Hum Res Ethics 2010;5:9-16.

31. Lemke AA, Wolf WA, Hebert-Beirne J, Smith ME. Public and biobank participant attitudes toward genetic research participation and data sharing. Public Health Genomics 2010;13:368-377.

32. Contopoulos-loannidis DG, Ntzani E, loannidis JP. Translation of highly promising basic science research into clinical applications. Am J Med 2003;114:477-484.

33. Ioannidis JP. Evolution and translation of research findings: from bench to where? PLoS Clin Trials 2006;1:e36.

34. Holland J, Baker SM. Customer participation in creating site brand loyalty. J Interact Marketing 2001;15:34-45.

35. Yarborough $M$, Sharp RR. Restoring and preserving trust in biomedical research. Acad Med 2002;77:8-14.

36. Bunin GR, Kazak AE, Mitelman O. Informing subjects of epidemiologic study results. Children's Cancer Group. Pediatrics 1996;97:486-491.

37. Dixon-Woods M, Jackson C, Windridge KC, Kenyon S. Receiving a summary of the results of a trial: qualitative study of participants' views. BMJ 2006;332:206-210.

38. Beskow LM, Burke W. Offering individual genetic research results: context matters. Sci Trans/ Med 2010;2:38cm20.

39. National Bioethics Advisory Commission. Research Involving Human Biological Materials: Ethical Issues and Policy Guidance, vol. 1. US Government Printing Office: Rockville, MD, 1999.

40. Bookman EB, Langehorne AA, Eckfeldt JH, et al.; NHLBI Working Group. Reporting genetic results in research studies: summary and recommendations of an NHLBI working group. Am J Med Genet A 2006;140:1033-1040.

41. Beskow LM, Linney KN, Radtke RA, Heinzen EL, Goldstein DB. Ethical challenges in genotype-driven research recruitment. Genome Res 2010;20:705-709.

42. Partridge $A H$, Winer EP. Informing clinical trial participants about study results. JAMA 2002;288:363-365.

43. Kreuter MW, Bernhardt JM. Reframing the dissemination challenge: a marketing and distribution perspective. Am J Public Health 2009;99:2123-2127.

44. Parker LS. Rethinking respect for persons enrolled in research. ASBH Exchange 2006;9:1,6-7. 